\title{
IAU South West and Central Asian Regional Office of Astronomy for Development
}

\author{
A. M. Mickaelian, S. A. Hakopian, S. V. Farmanyan, G. A. Mikayelyan \\ NAS RA V. Ambartsumian Byurakan Astrophysical Observatory (BAO), Armenia \\ E-mail: aregmick@yahoo.com
}

\begin{abstract}
The International Astronomical Union (IAU) announced its Strategic Plan on Astronomy for Development in 2009, during the International Year of Astronomy (IYA). One of its main components was the creation of the Office of Astronomy for Development (OAD) and corresponding Regional Offices (ROADs) for implementation and coordination of its aims. The OAD was created in Cape Town, South Africa and later on ROADs were created in 8 regions. Since 2015, Armenia hosts one of them, IAU South West Asian (SWA), later renamed to South West and Central Asian (SWCA) ROAD. At present, already 6 countries have officially joined (Armenia, Georgia, Iran, Kazakhstan, Tajikistan, and Turkey), but the Office serves for a rather broad region, from Eastern Europe to Central Asia. Armenia's geographical location and its historical role in astronomy (both for well-known archaeoastronomical heritage and the presence of the famous Byurakan Astrophysical Observatory (BAO) founded by Viktor Ambartsumian in 1946) serve as a link between Europe and Eastern Partnership countries, Middle East and Asia in general. We run activities in 3 directions, Task Forces (TF): TF1 Universities and Research, TF2 Children and Schools and TF3 Public Outreach. We present our projects and all other accomplishments and discuss the role of our ROAD in maintaining contacts and development of astronomy in the region, as well as contacts between Europe and the Eastern Partnership countries. Most up-to-date information about the IAU SWCA ROAD is available on its webpage at http://iau-swaroad.aras.am/eng/index.php.
\end{abstract}

Keywords: IAU - OAD - regional offices -astronomy development - astronomical education - public outreach.

\section{Introduction}

The International Astronomical Union (IAU) developed and adopted in 2009 a decadal Strategic Plan (SP) now entitled "Astronomy for Development". The SP significantly increases the role of astronomy for other sciences, technology, culture and society, as it is tightly linked to all this(Figure 1). This plan has been resulted from an extensive process of consultation beginning with a meeting of stakeholders in Paris in January 2008 and including feedback from key stakeholders during the 
various drafts of the SP. It was endorsed by the General Assembly of the IAU in Rio de Janeiro in August 2009, and builds on the momentum of the very successful International Year of Astronomy 2009 (IYA). The objective of this SP is to use Astronomy to stimulate development in all regions of the world. Crucial to the implementation of the SP was the creation of a global "Office of Astronomy for Development" (OAD). The OAD is tasked with establishing and strategically coordinating Regional Offices (ROADs) and Language Expertise Centres (LOADs) across the world as well as three Task Forces, namely (i) Astronomy for Universities and Research, (ii) Astronomy for Children and Schools, and (iii) Astronomy for the Public.

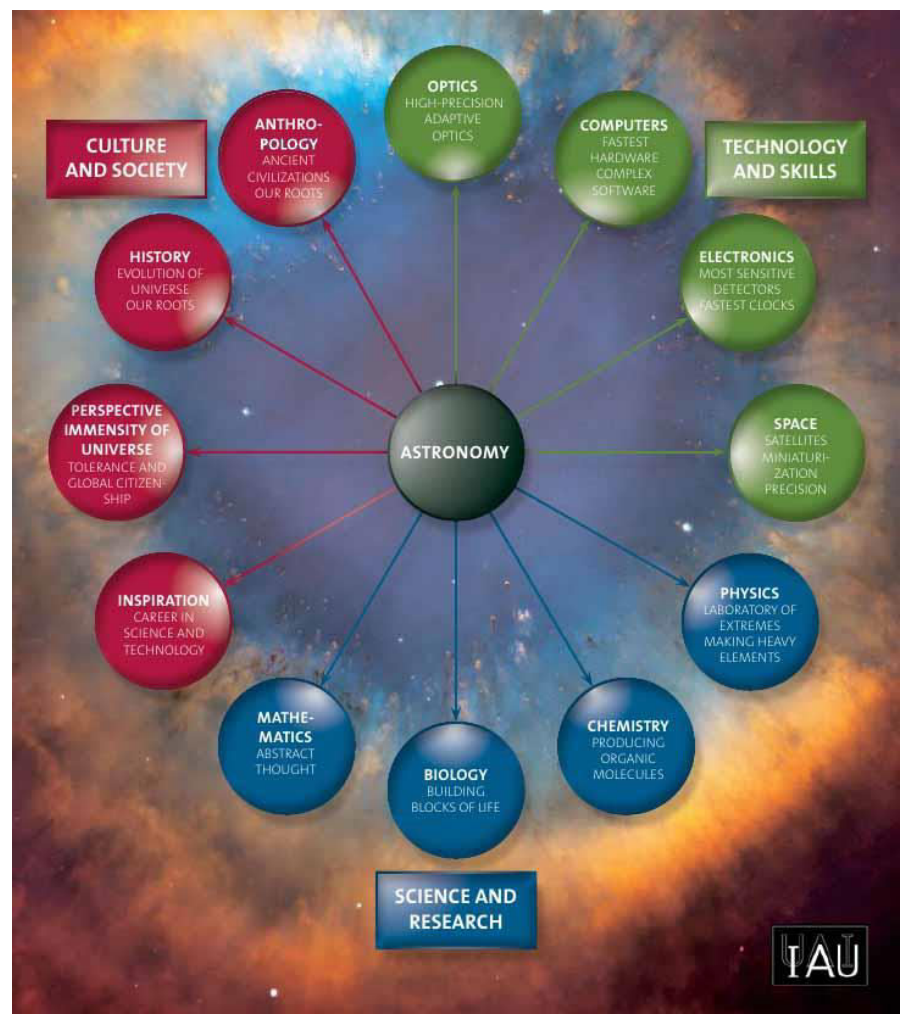

Astrophysics

Astrochemistry

Astrobiology

Astrogeology

Space research

Instrumentation

Astroinformatics

Astrostatistics

VO / e-Science

Astronomy in Culture

Archaeoastronomy

Astro tourism

Astro journalism

Astronomical

Education

Amateur Astronomy

Popular Astronomy

Figure 1. The relation of Astronomy with other sciences, technology, culture and society. A number of inter- and multi- disciplinary sciences and other fields are listed having tight relation to astronomy.

Through a strong partnership with the South African government, the OAD is hosted at the South African Astronomical Observatory (www.astro4dev.org), a facility of the National Research Foundation. Furthermore, 8 regional offices were created, one of them in Armenia. 


\section{The Establishment of the IAU SWA ROAD}

Armenia showed interest and activities in the establishment of one of the regional IAU offices since the beginning of the initiative (2009-2011). Armenia's proposal to host a ROAD was rather strong both from the point of view of the available facilities and ongoing activities. The proposal was finally approved on 10 June, 2015 and an agreement was signed between the IAU and the Byurakan Astrophysical Observatory (BAO) on 6 August 2015. The office was formally established on 1 September 2015 and was called IAU South West Asian(SWA) ROAD. Dr. Areg Mickaelian became its Director. SWA Regional Steering Committee was created in September 2015, as well as SWA webpage was opened also in September 2015 (http://iau-swa-road.aras.am, Figure 2). The staff members joined on 1 October 2015; Naira Azatyan (TF1 coordinator), Sona Farmanyan (TF3 coordinator), and Gor Mikayelyan (webmaster). Finally, the Inauguration Ceremony was held on 13 October 2015. IAU OAD / SWA ROAD Workshop was also attached to this event. Representatives from the IAU (General Secretary Piero Benvenuti, former Vice-President George Miley, OAD Director Kevin Govender) and representatives of regional and some other countries (Georgia, Iran, Turkey, Russia, Israel, Jordan) were present.

Like our long tradition to organize joint Armenian-Georgian (ByurakanAbastumani) workshops (colloquia) since 1974, we conducted a new series of Armenian-Iranian Astronomical Workshops (AIAW). The first one was organized on 13-16 October, 2015 in Byurakan, so that all Iranian guests were able to participate in the IAU SWA ROAD Inauguration Ceremony.
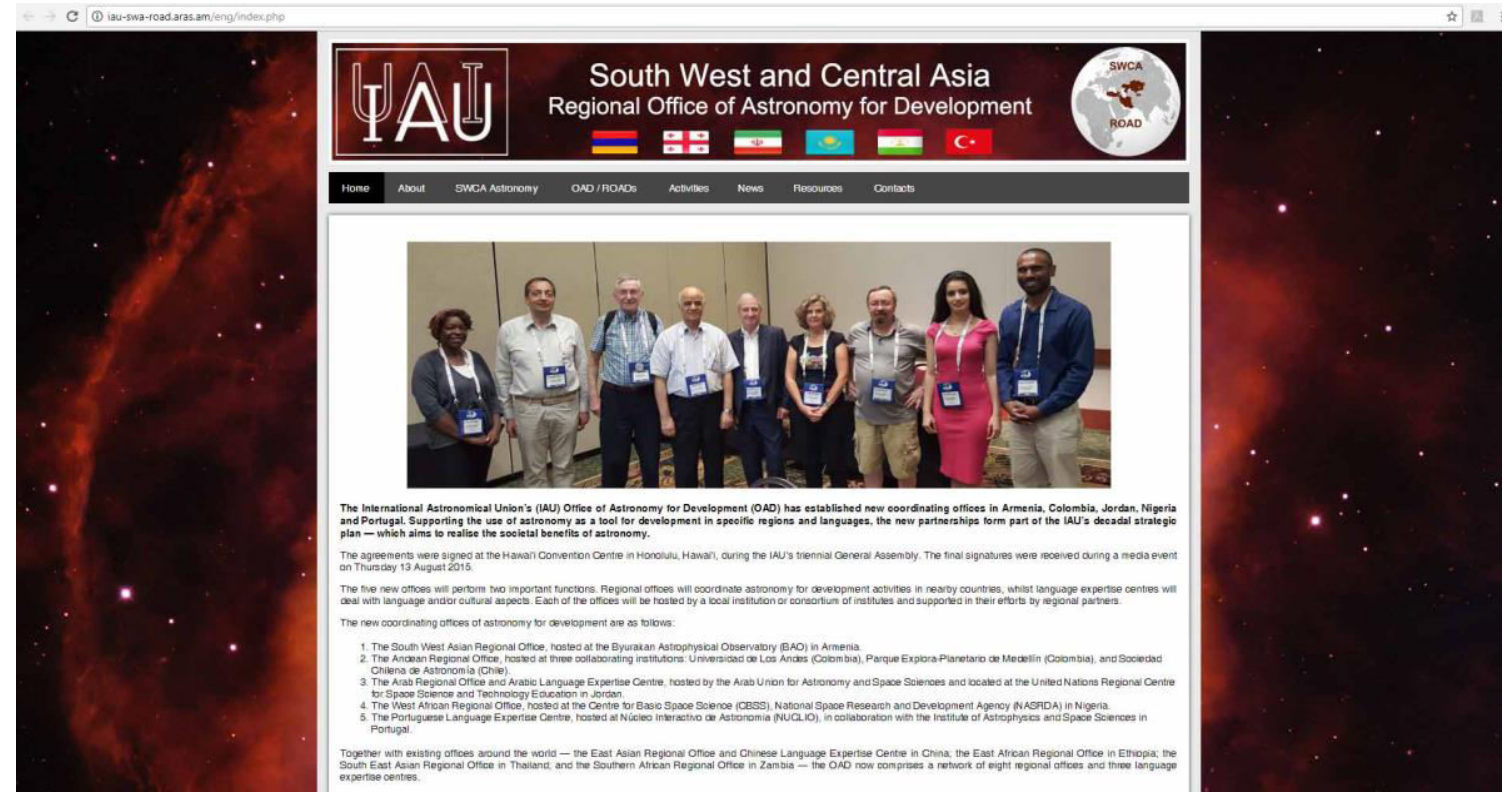

Figure 2. IAU South West and Central Asian ROAD webpage. 
Georgia and Iran were the first countries along with Armenia to officially join the SWA ROAD. During many years and centuries, these countries have had numerous relations in all areas, including science and Astronomy.

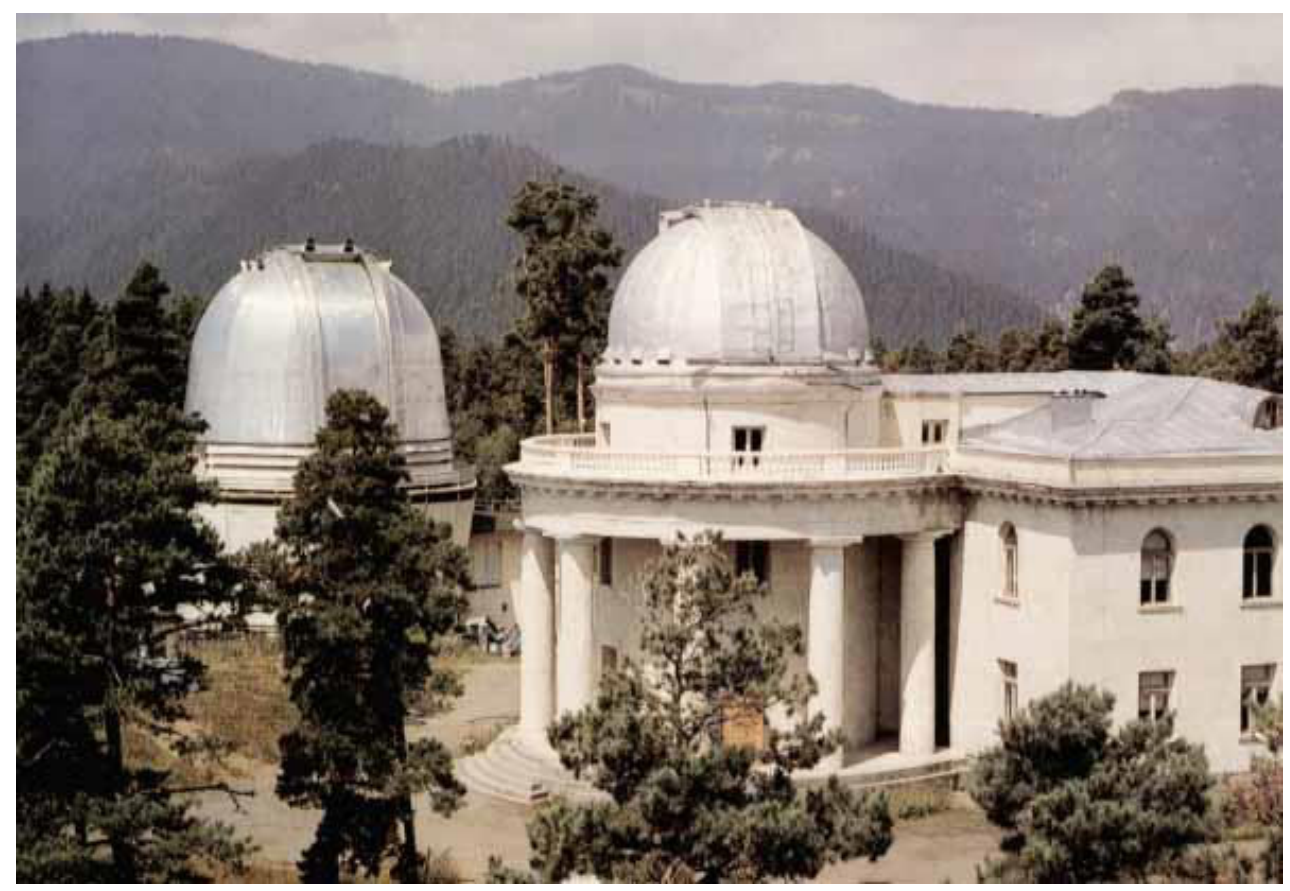

Figure 3. Abastumani Astrophysical Observatory in Georgia.

Astronomy in Georgia is generally represented in Abastumani Astrophysical Observatory (AbAO) founded in 1932. It is one of the leading scientific institutes in the country. Main fields of research are Solar System bodies (including near-Earth asteroids), various aspects of Solar physics, stellar astronomy (including binary stars and open clusters), extragalactic objects (AGNs), theoretical astrophysics, cosmology, atmospheric and Solar-terrestrial physics. Although research in these fields are carried out in other institutions in Georgia as well: Institute of Theoretical Physics at Ilia State University, Javakhishvili Tbilisi State University, School of Physics at Free University of Tbilisi. In AbAO, several telescopes are operational today, as well as the instruments for atmospheric studies. At present, in AbAO the 70-cm Maksutov meniscus telescope, 53-cm azimuthal reflector, 22-cm reflector ORI, 40-cm double astrograph, $53-\mathrm{cm}$ large and $11.5-\mathrm{cm}$ small solar coronagraphs are operational. They are equipped with CCD cameras. Spectroscopic and photometric observations are carried out. The instruments for atmospheric studies include: aerosol lidar $\mathrm{M}-10$ system, GRIPS-5 to measure the mezopause temperature, all-sky imager for measurements of some parameters of the nightglow, as well as the ozonometer. The new magnetometer to monitor the Earth's magnetic field has been installed recently. Astroclimatic conditions of Abastumani makes the location favourable for installation of advanced astronomical instruments, which is one of the main goals of the 
Observatory in the nearest future. In 2007 the Observatory was integrated with Ilia State University, merging scientific research and education which facilitated the growth of a new generation of researchers. There are groups of astronomers and astrophysicists in other Georgian universities and institutions as well. Georgian scientists collaborate with research centers and universities worldwide. Research groups participate in various international scientific projects. In the recent years the interest in astronomy in Georgia has been growing, which increases future perspectives of its development in the country.

Astronomy in Iran routes back to many thousand years ago. When Cyrus the Great, the founder of the Persian Empire, captured Babylon in 539 B.C., Magi who migrated there transformed Babylonian astronomy. They were the first to record planetary motion through constellations. In turn Magi learned from the Babylonian astronomers as well and translated Babylonian books into the early Persian language. Andromeda galaxy was first identified by a Persian astronomer Abd al-Rahman alSufi calling it a little cloud in his book of fixed stars around 964 . In $13^{\text {th }}$ century, Maragheh Observatory with a unique place in the history of Medieval Astronomy, was established, representing a new wave of scientific activities in the Islamic world. It had a key role in the development of sophisticated pre-Copernican non-Ptolemaic systems for explaining the planetary motions, and it was the model for several observatories that were built in Persia, Transoxiana, and Asia Minor up to the $17^{\text {th }}$ century. At present, research in Astronomy and Astrophysics has been conducted in a number of universities in Iran. There are two dedicated research institutes in Iran, IPM School of Astronomy in Tehranand Research Institute for Astronomy and Astrophysics in Maragheh (RIAAM). Among the most active universities in Astronomy one could name IASBS in Zanjan, Ferdowsi University of Mashhad (FUM), University of Tabriz, Shiraz, Birjand, Kerman and Zanjan universities, and Amirkabir University of Technology and Sharif University of Technology, both in Tehran. With nearly 400 members, the Astronomical Society of Iran (ASI) is a non-governmental organization that represents the Iranian community of astronomy internationally and with IAU, it coordinates various activities for professional and amateur astronomers and organizes training workshops, throughout the year. Iranian astronomers gather twice a year to share their ongoing research activities. The ASI also publishes an International Journal of Astronomy and Astrophysics (IJAA) which is a peer reviewed open access journal dedicated to original research and invited/review articles by distinguished astronomers around the world. Astronomy is also popular in Iranian schools. International Olympiad of Astronomy and Astrophysics (IOAA), in which 
Iranian teams do very well, see a participation of a few thousand students in a national exam. The Iranian National Observatory (INO, Figure 4) is under construction at an altitude of $3600 \mathrm{~m}$ at Gargash summit $300 \mathrm{~km}$ southern Tehran. The site selection was concluded in 2007 and the site monitoring activities have begun since then, which indicates high quality of the site with a median seeing of $0.7 \operatorname{arcsec}$ through the year. One of the major observing facilities of the observatory is a $3.4 \mathrm{~m}$ Alt-Azimuthal Ritchey-Chretien optical telescope which is currently under design. This $f / 11$ telescope will be equipped with high resolution medium-wide field imaging cameras as well as medium and high-resolution spectrographs.

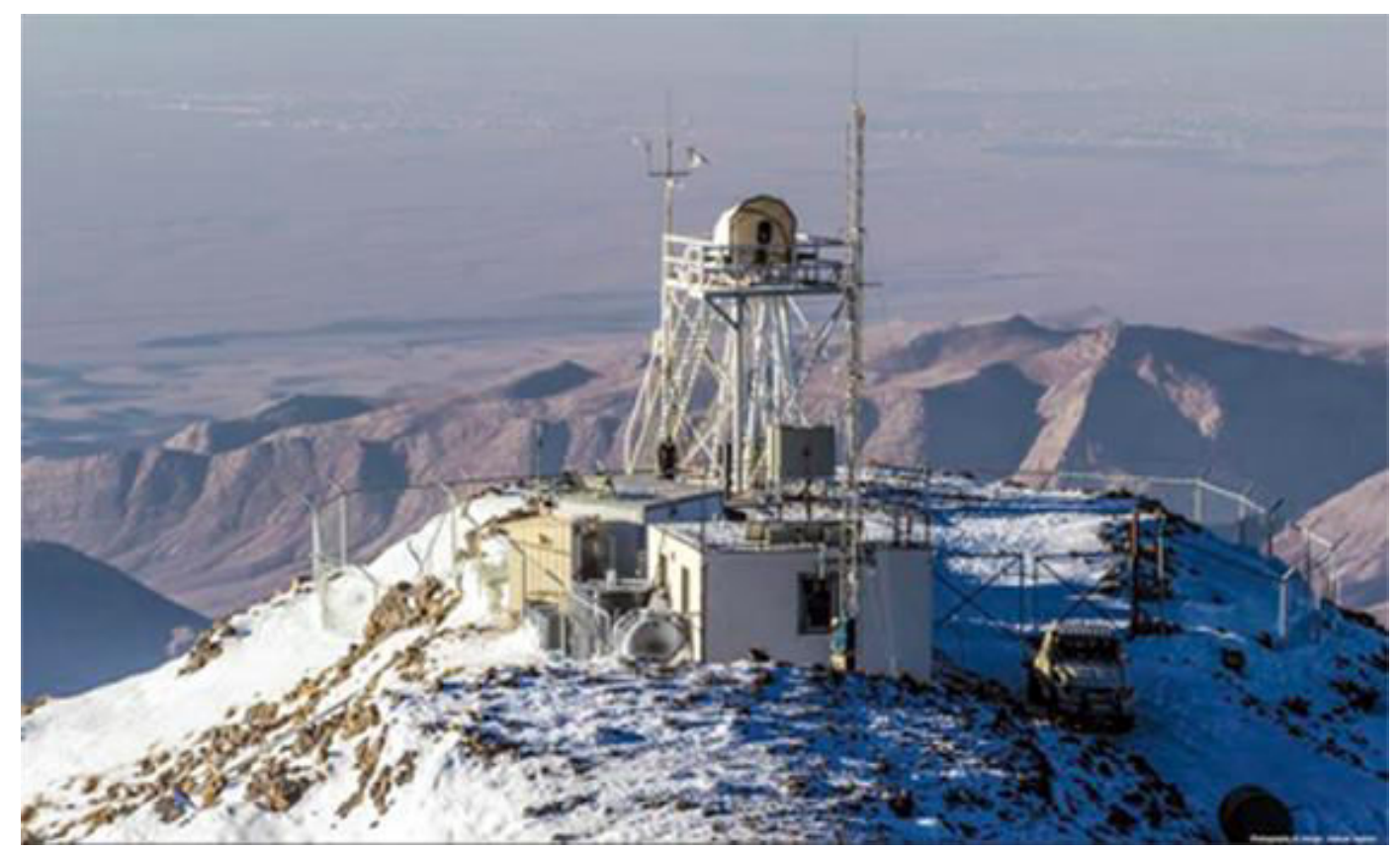

Figure 4. Iranian National Observatory (INO) at Gargash summit.

Since the beginning, IAU SWA ROAD developed activities in all three Task Forces. A number of meetings and schools were organized and a number of other projects were accomplished. Especially, successful were the activities related to Scientific Tourism in Armenia and in the region. We were awarded two grants in 2016: OAD grant for the development of Astro Tourism in the South West Asia and Swiss SDC grant for the development of Scientific Tourism in Armenia.

SWA ROAD representatives, the Director Areg Mickaelian and BAO Director Haik Harutyunian took part in the IAU Arab World ROAD opening inDecember 2015. In February-March 2016, Areg Mickaelian and Sona Farmanyan took part in the OAD/ROADs meeting in Cape Town, South Africa. The whole SWA team (Areg Mickaelian, Susanna Hakopian, the new TF1 coordinator, Sona Farmanyan, and Gor 
Mikayelyan) visited Georgia in March-April 2016 for tightening the collaboration and exchange of scientific knowledge. In August 2016, Areg Mickaelian and Sona Farmanyan were invited to RIAAM, Maragheh, Iran to participate in a workshop and gave talks.

We hold regular telecons both with OAD/ROADs andwith the SWA ROAD Steering Committee to give short reports on the accomplished affairs, and to discuss all current and future matters.

\section{Expansion to IAU SWCA ROAD}

In May 2016, Areg Mickaelian and the Scientific Secretary of BAO Elena Nikoghosyan visitedthree Central Asian countries, Uzbekistan, Kazakhstan and Tajikistan to recover our former contacts and collaboration. As a result, during June 2016, Kazakhstan and Tajikistan with official letters also joined our ROAD. This significantly strengthens our centre and expands its sphere of activities to Central Asia.

In Kazakhstan, Fesenkov Astrophysical Institute (APhI), Almaty (founded in 1941 ) is the main astronomical centre. At present Dr. RashitValiullin is the Director. One of the outstanding scientists, Prof. Eduard Denissyuk, was one of the first astronomers to spectroscopically observe Markarian galaxies and has long-year collaboration with BAO astronomers. There are three attached observatories to APHI: Kamenskoe Plateau Observatory (altitude 1450m, AZT-8 70cm, Zeiss-600 60cm, Hertz telescope-reflector $50 \mathrm{~cm}$, Wide aperture Maksutov meniscus telescope $50 \mathrm{~cm}$ ), TianShan Observatory (TShAO, altitude 2735m, two $1 \mathrm{~m}$ telescopes) and Assy-Turgen Observatory (altitude $2750 \mathrm{~m}, 1 \mathrm{~m}$ telescope). The research subjects are: physics of stars and nebulae; physics of the Moon and planets; cosmology, stellar dynamics \& computational astrophysics; nuclear astrophysics; artificial Earth satellites; advanced astrophysical research. Kazakh National University (KazNU) (with 16,000 students) is the main university preparing professional astronomers/astrophysicists.

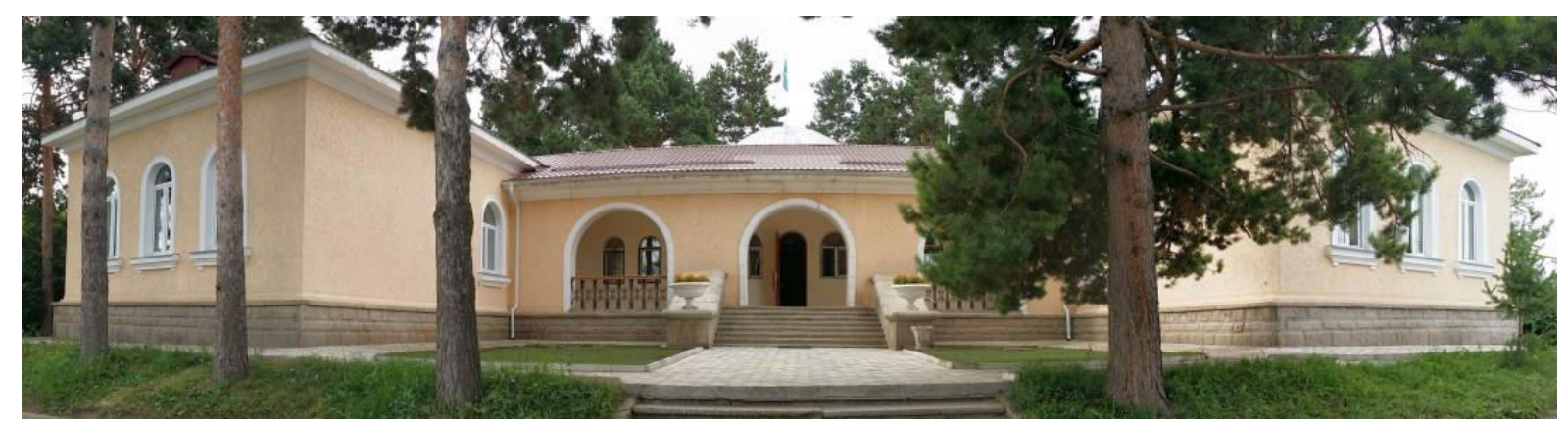

Figure 5. Main building of Fesenkov Astrophysical Institute in Almaty, Kazakhstan. 
In Tajikistan, Institute of Astrophysics of Academy of Science of the Republic of Tajikistan, in Dushanbe (founded in 1932) is the main astronomical centre. At present, Dr. Gulchehra Kokhirova is the Director. Profs. Pulat Babadjanov and Khursand Ibadinov, former directors and most eminent Tajik astronomers, are still active and strongly supported collaboration in frame of IAU ROAD as well. There are three attached observatories: Hissar Observatory (HisAO, altitude 730m, AZT-8, 40cm astrograph), the observatory "Sanglokh" (altitude $2300 \mathrm{~m}$ ) in Dangara area (1m telescope, $60 \mathrm{~cm}$ Carl Zeiss) and its branch, the observatory "Pamir" (altitude 4350m) in Murghab district of Badakhshan ( $70 \mathrm{~cm}$ telescope). Sanglokh Observatory was recently re-operated and the President of Tajikistan was present at the opening ceremony. The Institute's research subjects include: comets and asteroids, experimental astrophysics, meteor astronomy, ionospheric, astrometry, variable stars, structure and dynamics of stellar systems.

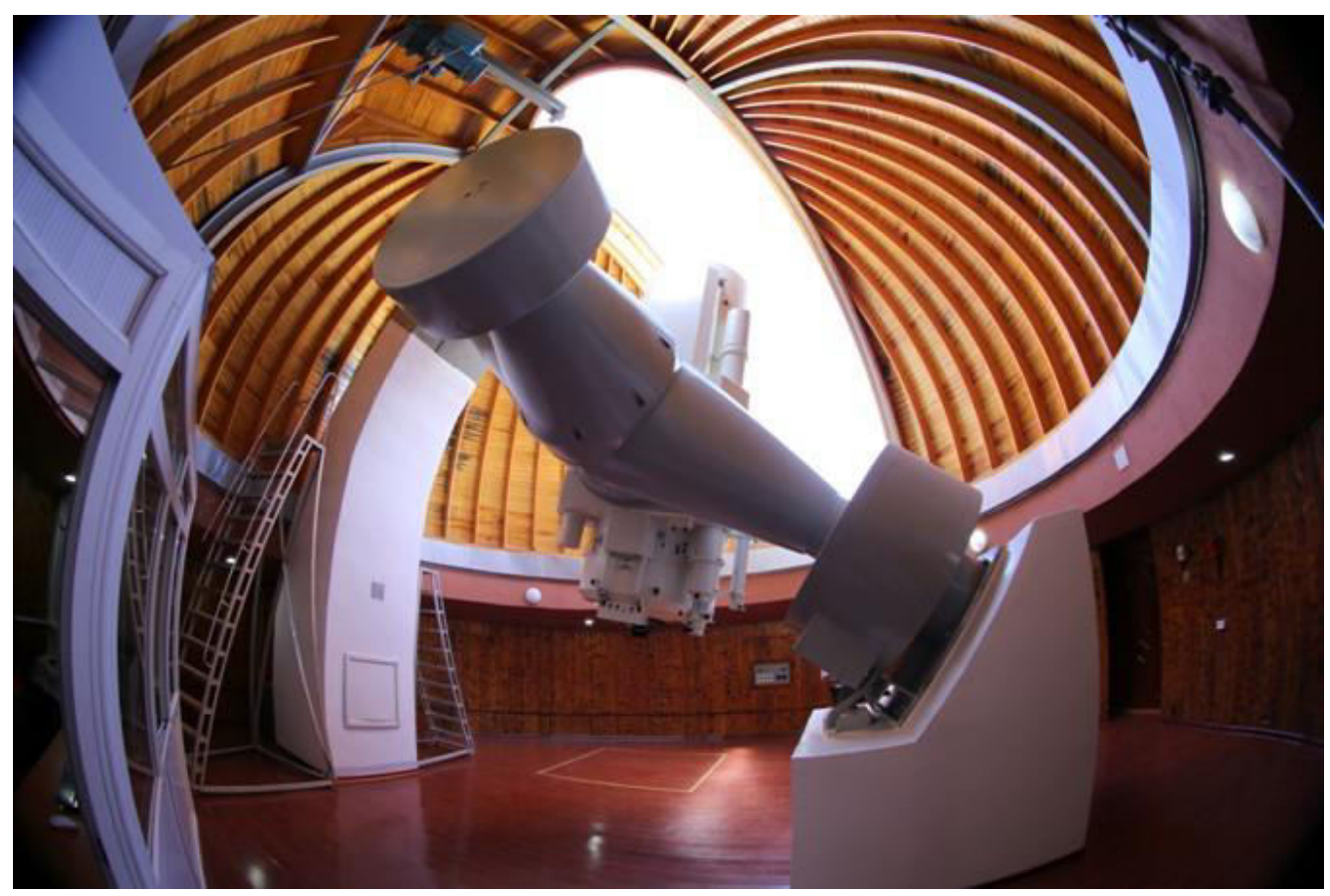

Figure 6. $1 \mathrm{~m}$ telescope of Sanglokh Observatory, Tajikistan.

Due to the involvement of Kazakhstan and Tajikistan, our regional centre was renamed to IAU South West and Central Asian (SWCA) ROAD. Corresponding changes and additions were done at our webpage.

On November 17, 2017, Turkey officially joined the South West and Central Asian ROAD office and expressed its desire to carry out its activities for the development of Astronomy by adopting Armenia's coordinating role. It is important 
to state that we also made a political big step. Armenia recognized as an astronomical centre by Turkey. Although it was set up by the IAU, however at present Turkey also accepts it and joins. Now our cooperation will be closer, visits and exchange of scientific experience more active. We hope to work on various astronomical topics to develop astronomy, to organize conferences, to carry out all the activities that this cooperation assumes.

\section{Other Collaborations}

Beside activities for the regional countries, IAU SWCA ROAD also encourages and strengthens collaboration with other regions and countries. Especially, promising are contacts with two neighbouring ROADs: East Asian (based in Beijing, China) and Arab World (based in Amman, Jordan). The first one is coordinating a huge region (China, Mongolia, North Korea) and is especially interested in the development of Silk Road projects that also relate Armenia. Arab World ROAD is in fact in the same big region (Middle East) and is our closest neighbour. We have discussed possibilities to tighter collaborate in all areas (TFs) and establish new programs.

In addition, recently we have discussed with Indian astronomers the possibilities to collaborate, as India is not a member of any ROAD and the closest one is SWCA.

\section{Projects and Other Activities}

It is a rule that both senior and young scientists from regional countries (especially Georgia and Iran) most often participate in our meetings, schools and other events. We also have many missions to the regional countries, again most often Georgia and Iran.

Armenia is rather active in organizing astronomical meetings, schools and other events. Among the most important meetings and schools held in Armenia, we would like to mention the IAU Symposia and Colloquia: IAU S029: Non-Stable Phenomena in Galaxies (1966), IAU S129: Observational Evidence of Activity in Galaxies (1986), IAU S137: Flare stars in Star Clusters, Associations and Solar Vicinity (1989), IAU S194: Activity in Galaxies and Related Phenomena (1998), IAU C184: AGN Surveys (2001), and IAU S304: Multiwavelength AGN Surveys and Studies (2013). Another large event was the all-European annual astronomical meeting in 2007, JENAM-2007(Joint European and National Astronomical Meeting), held in Yerevan, Armenia. It was the biggest ever scientific event in Armenia. Out of other meetings, 
one may mention joint meetings with a given country, namely Byurakan-Abastumani (Armenian-Georgian) Colloquia in 1974-2013, Armenian-French Workshops in 1995 and 2009, and the Armenian-Iranian Astronomical Workshop in 2015, as well as many meetings dedicated to the anniversaries of BAO, Viktor Ambartsumian, Anania Shirakatsi, Beniamin Markarian, Ludwik Mirzoyan and others. Especially many guests from the regional countries were present at BAO-70 anniversary meeting in September 2016. Our office has been awarded an OAD grant for organization of a Regional Summer School and Workshop in September 2018, and many regional guests are expected as well.

Among the summer schools, Byurakan International Summer Schools (BISS) are already very famous. We have started this initiative in 2006 and so far, have organized 5 such events: 1BISS in 2006, 2BISS in 2008, 3BISS combined with the IAU International School for Young Scientists (ISYA) in 2010, 4BISS in 2012, and 5BISS in 2016. 6BISS will be organized in September 2018. Byurakan Summer Schools for YSU students (BSS) are our local schools. They have been organized in 1995, 2005, 2009, and 2013. For the school students, on the initiative of Sona Farmanyan, we organize BAO Science Camps (BSC);4 such events were held in 2014-2017. ArAS School Astronomical Lectures program was started in 2012 on the initiative of Yervant Terzian and Areg Mickaelian. During 2012-2017, we have organized 4 such programs. In addition, in 2016 and 2017, we had "My Universe" contest and the winners visited $\mathrm{BAO}$.

Our ROAD representatives are rather active in participating in many international and regional meetings and have given numerous talks at such events related to our activities: scientific, educational, public, and in general about our SWCA ROAD.

In frame of the collaboration between Armenia and Iran, on 15-18 August 2016, Areg Mickaelian (IAU SWCA ROAD Director) and Sona Farmanyan (IAU SWCA ROAD TF3 Programme Coordinator) were invited to the Research Institute for Astronomy and Astrophysics of Maragha (RIAAM). During these days, the $8^{\text {th }}$ Advanced Astrophysics Workshop of Maragheh was organized. Areg Mickaelian delivered two lectures on "Astronomical Surveys, Catalogues, Databases, Archives, and Virtual Observatories" and "Multi-Wavelength Studies of Active Galaxies". Sona Farmanyan delivered two talks on"Archaeoastronomy and Cultural Astronomy in South West and Central Asia" and "Ancient Mythology and Cosmology". Sona also presented the IAU SWCA ROAD activities during 2015-2016.A number of discussions 
were held with Iranian astronomers, including Prof. Hossein Ebadie (RIAAM Director) and Prof. Pantea Davoudifar, with whom there is a collaboration in co-supervising of two Iranian students.

The fourth Middle East and African IAU Regional meeting (MEARIM IV), was jointly hosted by Entoto Observatory \& Research Center(EORC) and East African Regional Office of Astronomy for Development (ROAD-IAU) and was conducted from 22-25 May 2017 in Addis Ababa, Ethiopia with the theme of "Exploring our Universe for the benefit of Humankind". The purpose of the meeting was to strengthen and build capacity of Middle East and African Regional development in Astronomy, to provide a forum and network for scientists and students in the region to exchange ideas and experiences, share research outputs and knowledge, to brief and updates current status of Astronomy development in the region, to build joint working groups of research networks, to provide an opportunity for young and upcoming scientists in training, education and cross border co-supervision and sharing of resources and to put forward cooperation of the Middle East and African in furthering Astronomy and Space Science in the region. During the symposium there were activities such as scientific paper presentations, Plenary Guest and Invited speaker sessions, meetings and discussion on the general progress and Assessments of Astronomy and Astrophysics development in the Middle East and Africa Region. From Armenia, Areg Mickaelian (Director of BAO and SWCA ROAD) and Sona Farmanyan (Public Outreach Coordinator of SWCA ROAD) participated to the meeting and presented 5 talks on various subjects, including the one about SWCA ROAD.

The International Conference "Astronomical Heritage of the Middle East" approved by UNESCO Director General within UNESCO Participation Program for 2016-2017 was devoted to the role of Astronomy in Culture and other fields of human activities was held on 13-17 November, 2017 in Armenia(Figure 7). The conference brought together over 70 participants from 20 countries. A discussion of the problems of astronomy-related interdisciplinary sciences and further possible collaborations was organized. The Proceedings of the Meeting will be published by the Astronomical Society of the Pacific Conference Series(ASP CS), which is a worldwide known publication and the papers will appear in all libraries and electronic databases (including ADS).

We have given many presentations on our ROAD and its activities at a number of international meetings: NKAS (Belgrade, Serbia, 2015), IAU GA XXIX 
(Honolulu, Hawaii, USA, 2015), EWASS-2016 (Athens, Greece), EWASS-2017 (Prague, Czech Rep.) Armenia-Brandenburg Workshop (Nor Amberd, Armenia, 2017), MEARIM-IV (Addis Ababa, Ethiopia, 2017), etc.

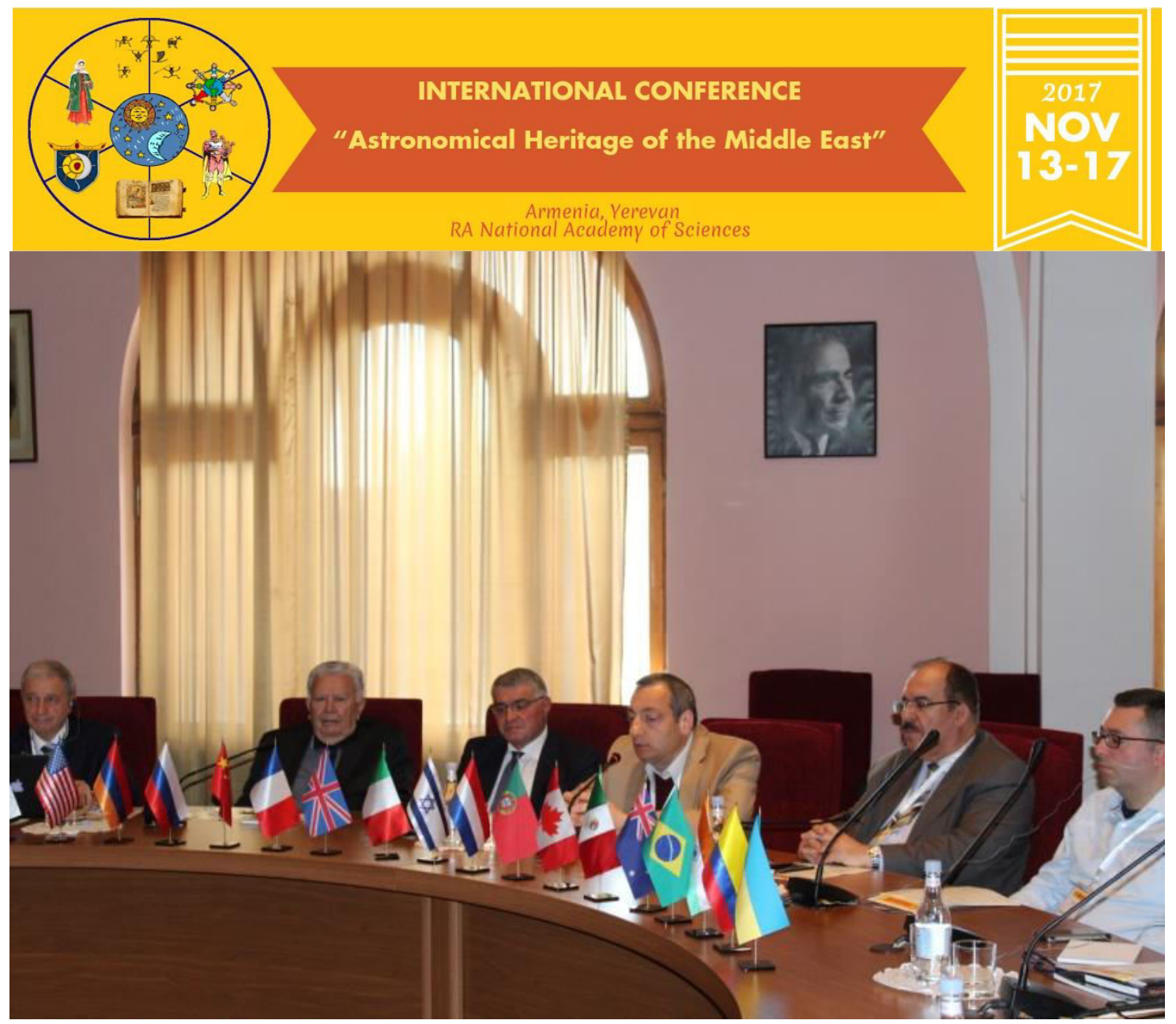

Figure 7. The International Conference "Astronomical Heritage of the Middle East".

We maintain a tight relation between the IAU SWCA ROAD and Eastern Europe. This includes collaboration with European countries, ArAS affiliation to the European Astronomical Society (EAS), ArAS collaboration with the Euro-Asian Astronomical Society (EAAS), Armenia's participation in South-East Regional European Astronomical Committee (SREAC), H2020 program (since 2016 Armenia is an associate member), collaboration between the Armenian Virtual Observatory (ArVO)and EuroVO, VO-France (also VO-Paris), GAVO, and VObs. We also have contacts with other European organizations; Euroscience, SEAC, etc. 


\section{Summary}

The status of the regional astronomical centre supports the regional and international contacts and collaboration both for Armenia and the neighboring countries; this way Armenia, by holding such a centre, strongly contributes to collaborations at all levels: professional, educational and popular. Astronomy in fact plays an important political role in establishing and strengthening friendship and cooperation of the regional nations, which is especially important in our complicated area.

Among our future plans, we envisage to enlarge the number of participating countries (involving more Central Asian and maybe some other ones), work harder for fundraising for implementation of various projects, organize regional meetings, workshops, schools and camps.

Acknowledgments. IAUSWCA ROAD team would like to thank IAU OAD and BAO Administration for supporting the office.

\section{References}

Mickaelian, A. M. 2017, Regional activities related to IAU strategic plan and integration of Armenia in European astronomy, Publ. Astron.Observ. of Belgrade (PAOB), Vol. 96, pp. 343-348

Mickaelian, A.; Azatyan, N.; Farmanyan, S.; Mikayelyan, G. 2016, IAU South West Asian ROAD // Astronomy in Focus, presented at the IAU XXIX General Assembly, 2015. Proc. IAU, Vol. 29A, Cambridge Univ. Press. (CUP), pp. 422-423

Mickaelian, A. M.; Hakopian, S. A.; Farmanyan, S. V.; Mikayelyan, G. A. 2018, IAU South West and Central Asian ROAD // Proc. Intern. Conf. "Astronomical Heritage of the Middle East", held on 13-17 November 2017, Astron. Society of the Pacific Conf. Series (ASP CS), in press

Mickaelian, A. M.; Khosroshahi, H. G.; Harutyunian, H. A. (Eds.) 2016, Proceedings of the Armenian-Iranian Astronomical Workshop, held on 13-16 October 2015 in Byurakan, Armenia // Yerevan, NAS RA "Gitutyun" Publishing House, p. 1-256 